\title{
Segmented Basophil Count
}

National Cancer Institute

\section{Source}

National Cancer Institute. Segmented Basophil Count. NCI Thesaurus. Code C135401.

The determination of the amount of segmented basophils present in a sample. 